\title{
Respiratory CARE and the Cochrane Collaboration
}

In this issue of Respiratory CARE, we are pleased to introduce a new feature to the Journal, The Cochrane Corner. The Cochrane Library is widely known for the collection of databases and the development of Cochrane Reviews through systematic reviews and meta-analyses. The goal of the Cochrane Reviews is to search the existing literature and synthesize the data to provide the basis for evidence-based medicine. As with any literature review, the goal is to identify well-conducted, randomized controlled trials as the cornerstone of the evidence.

Cochrane Reviews are extensive, detailed, methodological, systematic reviews and meta-analyses. These published reviews are often 50 pages or more and are accompanied by a shorter summary of findings and a plain-language synopsis of a couple pages. Full Cochrane Reviews are available by subscription, whereas plain language summaries are open access. Altruistically, the reviews are intended to improve practice and patient outcomes. Dissemination of the information is paramount.

In recent years, Cochrane Corners have been developed in collaboration with several journals to extend the reach of evidence to a wider audience. Respiratory CARE has entered into an agreement with Cochrane Rehabilitation to

Correspondence: Richard D Branson MSc RRT FAARC. E-mail: branson@aarc.org.

DOI: $10.4187 /$ respcare. 07800 publish a series of short summaries with commentary on topics of interest to our readers.

Each Cochrane Corner follows a standard format to present the evidence from the full Cochrane Review. An author chosen by the Journal staff provides commentary highlighting the importance of the Cochrane

See the Related Article on Page 420

Review to patient care and the profession. Our first Cochrane Corner, in this issue, reviews the evidence regarding the use of noninvasive ventilation and CPAP in the treatment of acute cardiogenic pulmonary edema, and is written by Dean Hess. ${ }^{1}$ In a subsequent issue, Denise Willis (Editorial Intern for REsPIRATORY CARE) will comment on the role of NIV in cystic fibrosis. ${ }^{2}$

The Editorial Office at Respiratory CARE is pleased to offer this new feature to our readers and looks forward to our continued collaboration with Cochrane Rehabilitation.

Richard D Branson Editor-in-Chief, REsPIRATORY CARE Department of Surgery University of Cincinnati Cincinnati, Ohio

\section{REFERENCES}

1. Hess DR. Should noninvasive ventilation be used for treatment of acute cardiogenic pulmonary edema? A Cochrane Review summary with commentary. Respir Care 2020;65(4):573-574.

2. Willis LD. The role of noninvasive ventilation in cystic fibrosis. A Cochrane Review summary with commentary. Respir Care, in press. 